\title{
Growth inhibitory efficacy of natural products in a model for triple negative molecular subtype of clinical breast cancer (Review)
}

\author{
NITIN TELANG \\ Cancer Prevention Research Program, Palindrome Liaisons Consultants, Montvale, NJ 07645-1559, USA
}

Received July 18, 2017; Accepted July 27, 2017

DOI: $10.3892 /$ br.2017.958

\begin{abstract}
Global gene expression profiling identifies predictive and prognostic biomarkers and rationalizes breast cancer subtype-targeted treatment. The Anthracyclin/Taxol and survival pathway specific small molecular inhibitors, constitute current treatment options. These options are associated with acquired tumor resistance and emergence of drug-resistant cancer stem cells. Dietary supplements and constitutive bioactive phytochemicals with relatively low systemic toxicity may provide testable alternatives for current therapy. Human breast epithelial cell lines 184-B5 (non-tumorigenic triple negative cell type) and MDA-MB-231 (breast carcinoma derived triple negative cell type) were used as the experimental models. Putative cancer chemo-preventive natural products and their constitutive bioactive agents represented the test agents. Anchorage independent growth, cell cycle progression and cell apoptosis quantified the treatment efficacy. Compared to the 184-B5 cells, the MDA-MB-231 cells exhibited anchorage-independent growth indicative of persistent cancer risk. Additionally, the MDA-MB-231 cells exhibited hyper-proliferation, accelerated cell cycle progression and inhibited apoptosis indicative of loss of homeostatic growth control. The test agents inhibited anchorage-independent growth via cytostatic and pro-apoptotic effects. The triple negative carcinoma-derived Doxorubicin-resistant phenotype exhibited cancer stem cell markers, including tumor spheroid formation and expression of CD44, NANOG and c-Myc. These data identify clinically relevant mechanistic leads for the efficacy of natural products in the aggressive therapy-resistant breast cancer subtype and suggests a testable approach for cancer stem cell-targeted therapy.
\end{abstract}

Correspondence to: Dr Nitin Telang, Cancer Prevention Research Program, Palindrome Liaisons Consultants, 10 Rolling Ridge Road, Montvale, NJ 07645-1559, USA

E-mail: entitytoo@gmail.com; ntelang3@gmail.com

Key words: natural product, clinical breast cancer

\section{Contents}

1. Introduction

2. Experimental models

3. Mechanistic biomarkers

4. Natural products

5. Efficacy of natural products

6. Conclusions and future directions

\section{Introduction}

Metastatic breast cancer is a prevalent cause of mortality in the US population. The American Cancer Society has projected approximately 246,660 newly diagnosed invasive breast cancer cases and approximately 40,450 breast cancer-related deaths in women in 2017 and the life time breast cancer risk is estimated at 1 in 8 individuals (1). These actuarial data emphasize a need to identify specific and sensitive prognostic and predictive biomarkers and efficacious cancer subtype specific-targeted therapy.

Conventional chemo-endocrine therapeutic options for clinical breast cancer are based on the status of hormone receptors, proliferation and cell apoptosis. Additionally, global profiling of differentially expressed genes has facilitated the molecular/genetic classification of breast cancer subtypes, and thus, has provided rational subtype-based treatment options (2).

The Luminal A molecular subtype is classified as estrogen receptor (ER)-positive, progesterone receptor (PR)-positive and human epidermal growth factor receptor-2 (HER-2)-negative breast cancer. The Luminal B subtype is characterized by the presence of all three abovementioned receptors. The treatment options for the two subtypes include the use of selective ER modulators and aromatase inhibitors, with or without HER-2 targeted therapy. The triple negative molecular subtype is classified as ER-negative, PR-negative and HER-2-negative breast cancer which responds only to conventional chemotherapy and select cellular signaling pathway specific small molecular inhibitors (2-5).

Current long-term treatment options for conventional chemo-endocrine therapy and for small molecular based-targeted therapy are frequently associated with acquired tumor resistance due to the emergence of drug-resistant stem cell population. These limitations compromise therapeutic efficacy and promote drug-resistant disease progression $(3,4,6)$. 
Preclinical studies on human tissue derived cell culture models may facilitate investigations directly on the target tissue to reduce extrapolation and enhance clinical translatability, thereby, providing facile in vitro experimental approaches that are complementary to conventional in vivo approaches $(3,4)$.

Natural products such as dietary supplements, phytochemicals and herbal formulations have been extensively used either as palliative or adjuvant treatment options for breast cancer patients in complementary and alternative medicine (7-9). These agents, due to their inherently minimal toxicity, may provide testable alternatives for limitations of conventional chemo-endocrine and small molecular-targeted therapeutic options. Previously published data on cell culture models for Luminal A, HER-2 enriched and triple negative molecular subtypes of clinical breast cancer have demonstrated that several mechanistically distinct natural phytochemicals and herbal extracts function as cytostatic and pro-apoptotic agents conferring potent growth inhibitory efficacy in these clinically relevant model systems (10-21).

The present review summarizes experimental evidence identifying novel mechanistic leads for the efficacy of select natural products and their respective constituent bioactive components in the clinically relevant cell culture model for triple negative breast cancer, and provides experimental evidence supporting the development of a drug-resistant cancer stem model. Furthermore, this review suggests a testable approach for cancer stem cell-targeted therapeutic options for chemoendocrine therapy-resistant aggressive breast cancer.

\section{Experimental models}

184-B5 model. This model was derived from a human reduction mammoplasty specimen. The cells were ER-, $\mathrm{PR}^{-}$and HER-2- and non-tumorigenic (22). This cell line served as the baseline control for quantitative growth parameters.

MDA-MB-231 model. The triple negative molecular subtype of clinical breast cancer lacks the expression of ER, PR and HER-2 (2). The human mammary carcinoma-derived MDA-MB-231 cells lack the expression of the three receptors $(23,24)$. This cell line represents a model for the triple negative breast cancer subtype.

\section{Mechanistic biomarkers}

Growth parameters. Conventional quantitative parameters such as population doubling time and saturation density have been widely used as endpoints to quantify growth. Population doubling time is monitored during the four-day exponential growth phase by determining cell viability. The data are expressed as arithmetic mean from the four time points. Saturation density is determined by viable cell number at day 7 post-seeding (16-18).

Anchorage-independent growthassay. Anchorage-independent growth is a sensitive and specific in vitro surrogate endpoint for the tumorigenic potential of carcinoma-derived cell lines (12-15,19-21). For this assay, 184-B5 and MDA-MB-231 cell lines at a predetermined cell density were suspended in $0.33 \%$ agar and overlaid on a basement layer of $0.6 \%$ agar.
Table I. Natural product test agents.

\begin{tabular}{ll} 
Test agent & \multicolumn{1}{c}{ Natural source } \\
\hline Dietary supplements & \\
OPE & Citrus fruits, limonoids \\
RME & Rosemary leaves, phenolic \\
& terpenoids \\
GTE & Green tea leaves, polyphenols
\end{tabular}

Constitutive bioactive phytochemicals

$\begin{array}{ll}\text { UA } & \text { Tri-terpenoid } \\ \text { EGCG } & \text { Tea polyphenol }\end{array}$

OPE, orange peel extract; RME, rosemary extract; GTE, green tea extract; UA, ursolic acid; EGCG, epigallocatechin gallate.

The anchorage-independent colony counts were performed at day 21 post-seeding.

Cell cycle progression and cell apoptosis. The parameters were quantified by flow cytometry-based fluorescence-assisted cell sorting. Cell populations in the $\mathrm{G}_{1}, \mathrm{~S}, \mathrm{G}_{2} / \mathrm{M}$ and sub $\mathrm{G}_{0}$ phases of cell cycle were then monitored. The data are expressed as $\mathrm{G}_{1}: \mathrm{S}+\mathrm{G}_{2} / \mathrm{M}$ ratio, $\mathrm{S}+\mathrm{G}_{2} / \mathrm{M}$ :sub $\mathrm{G}_{0}$ ratio, and \% sub $\mathrm{G}_{0}$ population $(17,19-21)$.

Drug-resistant cancer stem cells. Drug-resistant phenotype was isolated from a subpopulation of Doxorubicin-resistant (DOX-R) MDA-MB-231 cells. To isolate the DOX-R phenotype, MDA-MB-231 cells were treated with $0.5 \mu \mathrm{M}$ DOX (maximum cytostatic concentration) for 7 days. The surviving cell population was expanded in the presence of $0.5 \mu \mathrm{M}$ DOX for at least 5 passages prior to the experiments. The DOX-R cells were monitored for the status of stem cell-specific markers, including tumor spheroid formation, and expression of stem cell-specific molecular markers CD44, NANOG and c-Myc. The spheroid formation was monitored by tumor spheroid colony counts in serum-free culture conditions on day 14 post-seeding of 100 drug-resistant cells in ultralow adherence culture plates. The expression of stem cell-specific molecular markers CD44, NANOG and c-Myc was monitored by fluorescence-assisted cell sorting of DOX-R cells stained with specific fluorescein isothiocyanate (FITC)-labeled antibodies according to the optimized protocol provided by the vendors. These data were then normalized to FITC-IgG, and expressed as log mean fluorescence units (17).

\section{Natural products}

Non-fractionated extracts from dietary natural products, as well as their constitutive bioactive phytochemicals were selected as the test agents based on the evidence supporting cancer chemo-preventive efficacy in the animal models for organ site cancer (16-18). The test agents used in the experiments are presented in Table I.

Dose response experiments were utilized to identify maximum cytostatic concentrations $\left(\mathrm{IC}_{90}\right)$. The maximum 
Table II. Status of homeostatic growth control in triple negative MDA-MB-231 cells.

\begin{tabular}{llc}
\hline & \multicolumn{2}{c}{ Relative to 184-B5 cells ${ }^{\mathrm{a}}$} \\
\cline { 2 - 3 } Biomarker & \multicolumn{1}{c}{ Range } & Median \\
\hline Population doubling & -53.6 to $-55.9 \%$ & $-54.7 \%$ \\
Saturation density & +47.5 to $+50.2 \%$ & $+48.8 \%$ \\
$\mathrm{G}_{1}: \mathrm{S}+\mathrm{G}_{2} / \mathrm{M}$ & -70.5 to $-73.9 \%$ & $-72.2 \%$ \\
$\mathrm{~S}+\mathrm{G}_{2} / \mathrm{M}:$ Sub $\mathrm{G}_{0}$ & +8.5 to $+9.5 \mathrm{X}$ & $+9.0 \mathrm{X}$ \\
Anchorage-independent & & \\
colony formation & $+95 \%$ to $+100 \%$ & $+97.5 \%$ \\
\hline
\end{tabular}

${ }^{\mathrm{a}}$ Extrapolated from multiple data points $(18,19,21)$.

cytostatic concentration was defined as the highest concentration of the test compound that produces a viable cell number that is equal to the initial seeding density. The concentration producing viable cell number that was lower than the initial seeding density was considered toxic. These pre-determined maximum cytostatic concentrations of the test compounds were used in the experiments to evaluate their growth inhibitory effects and mechanistic efficacy.

\section{Efficacy of natural products}

Status of homeostatic growth control in triple negative model. To identify growth advantage of the model, growth parameters of tumorigenic ER', PR-and HER-2- MDA-MB-231 (triple negative) cells were compared against the non-tumorigenic ER', PR and HER-2- 184-B5 (triple negative) cells, representing the baseline control. These data demonstrated that relative to 184-B5 cells, the triple negative tumor-derived cell line exhibits a $54.7 \%$ decrease in population doubling, and a $48.8 \%$ increase in saturation density (Table II). Additionally, this cell line exhibits a $72.2 \%$ decrease in $\mathrm{G}_{1}: \mathrm{S}+\mathrm{G}_{2} / \mathrm{M}$ ratio due to aberrant hyper-proliferation and a 9-fold increase in the $\mathrm{S}+\mathrm{G}_{2} / \mathrm{M}: \mathrm{Sub} \mathrm{G}_{0}$ ratio due to downregulated cell apoptosis. It is also noteworthy that unlike the non-tumorigenic control cells, the tumor-derived cells exhibit anchorage-independent growth as demonstrated by a substantial $97.5 \%$ increase in the number of anchorage-independent colonies. Collectively, these data demonstrate that the tumorigenic cell line that is hyper-proliferative, less apoptotic and exhibits anchorage-independent growth, displays loss of homeostatic growth control and persistent cancer risk. Furthermore, these growth properties of human tumorigenic cells, which are distinct from those of non-tumorigenic human cells provide evidence to support a clinical relevance for the experimental models $(16,18-21)$.

Efficacy of natural products and constitutive bioactive phytochemicals in triple negative model. The triple negative subtype lacks the expression of hormone and growth factor receptors and therefore is resistant to endocrine therapy and to HER-2-targeted therapy $(2,4,5)$. The response of the triple negative subtype to cytotoxic chemotherapy and to molecular pathway-targeted therapy is associated with acquired tumor
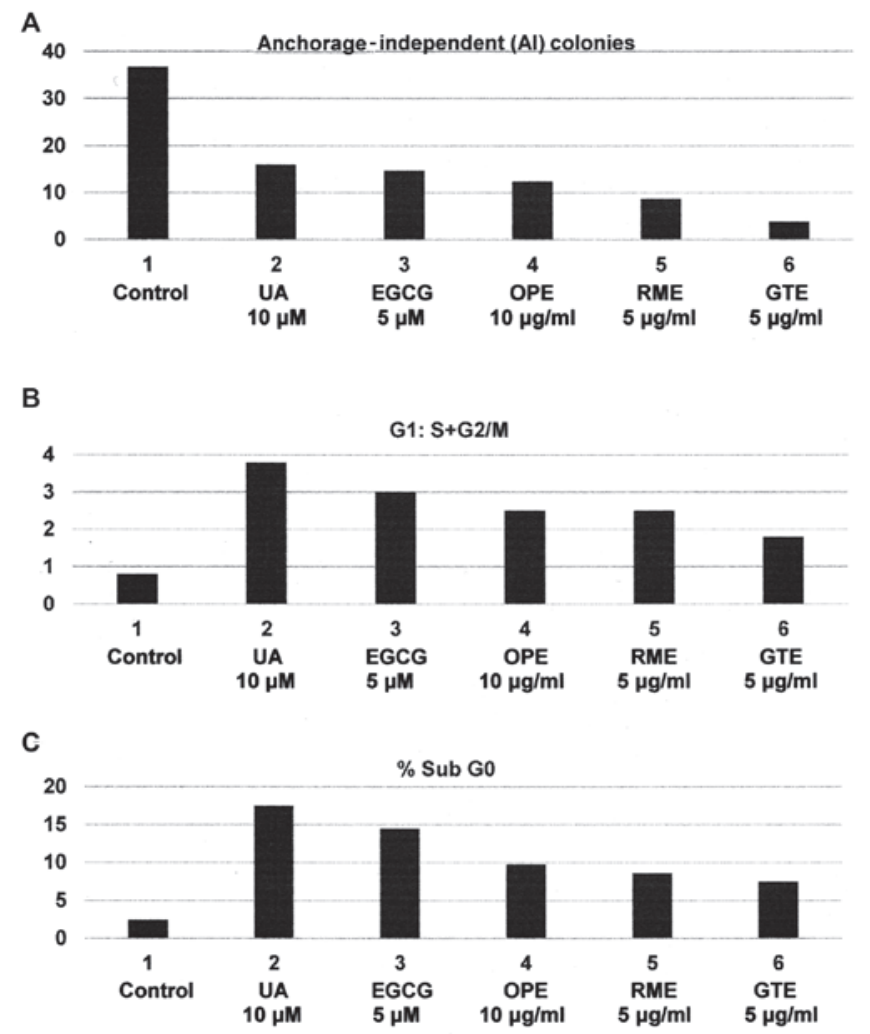

Figure 1. Efficacy of natural products and constitutive bioactive agents in triple negative MDA-MB-231 cells: (A) Inhibition of anchorage-independent colony formation. Treatment with respective maximum cytostatic concentrations of constitutive bioactive agents UA and EGCG, and dietary supplements OPE, RME and GTE. Colony counts at day 21 post-seeding of 1,000 cells. Control > UA, EGCG, OPE, RME and GTE (one-way ANOVA) and Dunnett's multiple comparison test $(\alpha=0.05)$. (B) Increase in $G_{1}: S+G_{2} / M$ ratio. Cells treated for $24 \mathrm{~h}$ with maximum cytostatic concentrations of constitutive bioactive agents and dietary supplements. Cell cycle analysis by flow cytometry. Control < UA, EGCG, OPE, RME and GTE. One-way ANOVA and Dunnett's multiple comparison test $(\alpha=0.05)$. (C) Induction of cell apoptosis. Cells treated for $24 \mathrm{~h}$ with maximum cytostatic concentrations of constitutive bioactive agents and dietary supplements. Quantification of cells in the sub $\mathrm{G}_{0}$ (apoptotic) phase by flow cytometry. Control < UA, EGCG, OPE, RME and GTE. One-way ANOVA and Dunnett's multiple comparison test $(\alpha=0.05)$. UA, ursolic acid; EGCG, epigallocatechin gallate; OPE, orange peel extract; RME, rosemary extract; GTE, green tea extract.

resistance and emergence of drug-resistant cancer stem cells $(4-6,25,26)$. The experiments presented in Fig. 1A-C were designed to examine the growth inhibitory effects of dietary supplements orange peel extract (OPE), rosemary extract (RME), green tea extract (GTE) and their constitutive bioactive agents ursolic acid (UA) and epigallocatechin gallate (EGCG) on the triple negative MDA-MB-231 model. The test agents inhibited anchorage-independent colony formation at their respective maximum cytostatic concentrations (Fig. 1A). The cytostatic effect of these test agents on cell cycle progression was associated with $\mathrm{G}_{1}$ arrest, leading to an increased $\mathrm{G}_{1}: S+\mathrm{G}_{2} / \mathrm{M}$ ratio (Fig. $1 B$ ). Increased $\mathrm{G}_{1}: \mathrm{S}+\mathrm{G}_{2} / \mathrm{M}$ ratio occured predominantly due to $\mathrm{G}_{1}$ arrest and the resultant inhibition of $G_{1}$ to $S$ phase transition, leading to investigation on the status of $\mathrm{G}_{1}$-specific cyclins and other relevant signaling proteins. Additionally, the pro-apoptotic effect was evidenced by increases of cell population in apoptotic sub $\mathrm{G}_{0}$ phase of the cell cycle (Fig. 1C). The observed increase 
A

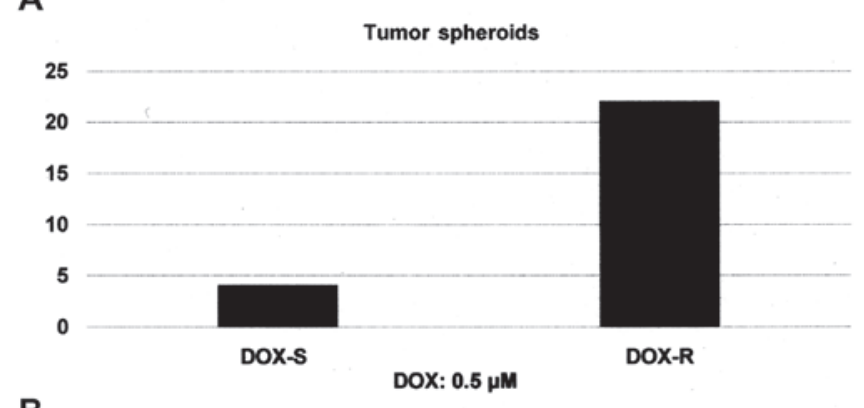

B

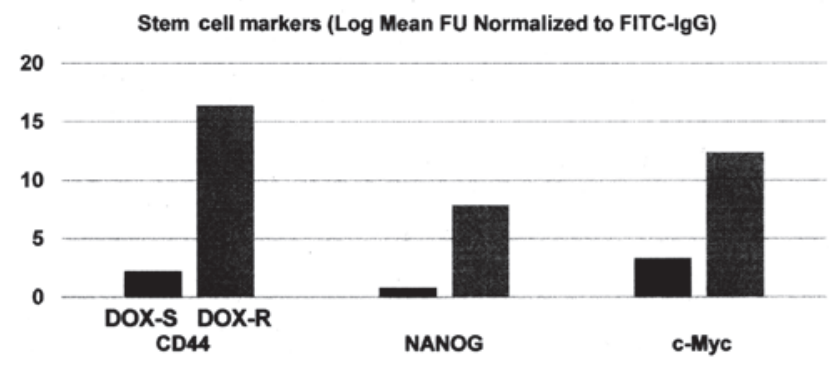

Figure 2. Cancer stem cells in triple negative model: (A) Tumor spheroids in triple negative MDA-MB-231 cells. DOX-S and -R cells were seeded in ultra low attachment plates at a seeding density of 100 cells in the presence of $0.5 \mu \mathrm{M}$ DOX. Tumor spheroid counts at day 14 post-seeding. DOX-S vs. DOX-R P=0.02 (two-sample t-test). (B) Expression of stem cell markers in DOX-S and DOX-R cells. Log mean fluorescence units. DOX-S vs. DOX-R CD44, $\mathrm{P}=0.02$; NANOG, $\mathrm{P}=0.01$; c-Myc $\mathrm{P}=0.03$ (two-sample t-test). DOX, doxorubicin.

in the sub $\mathrm{G}_{0}$ population leading to investigation on the status of apoptosis-related proteins. The statistical significance of these data were analyzed by one-way analysis of variance (ANOVA) and Dunnett's multiple comparison test $(\alpha=0.05)$. Collectively, these data provide mechanistic leads to identify potential molecular targets responsible for the efficacy of relatively non-toxic naturally occurring agents and suggest testable alternative to therapy-resistant triple negative breast cancer $(16,18,27)$.

Several mechanistically distinct natural products have exhibited growth inhibitory efficacy in HER-2 expressing human mammary epithelial cells that represent a cellular model for HER-2 enriched breast cancer. The molecular mechanisms responsible for the anti-proliferative and pro-apoptotic efficacy of these agents include $G_{1}$ arrest and inhibition of $G_{1}$-specific cyclins and modulation of apoptotic-specific proteins $(16,18)$. Additionally, chemo-preventive agents including resveratrol, retinoids and rosemary terpenoids exhibit effective inhibition of inducible cyclo-oxygenase-2 (COX-2) activity in this model (28-31).

In the current context it is noteworthy that in the triple negative subtype, the tumor suppressive function of the $R B$ gene is frequently compromised $(32,33)$. Thus, the CyclinD-CDK4/6-pRB pathway has been exploited as a therapeutic target $(34,35)$. Additionally, the present cellular model for triple negative breast cancer subtype harbors a gain-of-function R280K mutation in the tumor suppressor TP53 gene that confers survival advantage via the upregulation of cell migration and invasion (36). Thus, the present status of TP53 may be an additional therapeutic target.
Nutritional herbs are commonly used in herbal formulations for the treatment of breast cancer patients $(7-9,37)$. Thus, preliminary evidence suggests that in the present triple negative model, nutritional herbs Tabebiua avellandae, Cornus officinalis and Dipsacus asperoides confer growth inhibitory effects via the RB pathway, and pro-apoptotic effects via the intrinsic mitochondrial BAX and caspase pathways (19-21).

Cancer stem cells in triple negative model. Acquired tumor resistance to chemo-endocrine therapy is associated with the emergence of drug-resistant stem cells $(4,6)$. Furthermore, acquired resistance to small molecular inhibitors of PI3K/AKT/mTOR pathways in the triple negative subtype $(4,5,25)$, emphasizes investigations focused on a therapy-resistant stem cell population.

Select nuclear transcription factors Oct-3/4, Klf4, Sox 2 and c-Myc (Yamanaka factors) are expressed in cancer stem cells derived from multiple organ sites (38-43). These transcription factors are also essential for the induction of pluripotent stem cells derived from adult somatic cells (44-47). The experiments presented in Fig. 2A and B were designed to examine the status of select stem cell markers in DOX-R MDA-MB-231 phenotype. Relative to the drug sensitive phenotype, the DOX-R phenotype exhibited a 4.4-fold increase $(\mathrm{P}=0.02)$ in the number of tumor spheroids (Fig. 2A). Additionally, in the DOX-R phenotype the expression of stem cell-specific cellular marker $\mathrm{CD} 44$ exhibited a 6.4 -fold increase $(\mathrm{P}=0.02)$, while the molecular markers NANOG exhibited an 8.8 -fold increase $(\mathrm{P}=0.01)$ and $\mathrm{c}-\mathrm{Myc}$ exhibited a 2.7 -fold increase $(\mathrm{P}=0.03)$, relative to the DOX-S phenotype (Fig. 2B). These data were analyzed for their statistical significance by the two-sample t-test. Collectively, these data on the status of stem cell marker expression characterize the DOX-R stem cell model, thereby validating an experimental approach for the evaluation of stem-targeted therapeutic efficacy.

Ongoing research directions include experiments designed to examine the efficacy of promising natural products on the developed triple negative breast cancer stem cell model. These studies are expected to provide evidence for effectivetargeted intervention directly on therapy-resistant breast cancer stem cells.

\section{Conclusions and future directions}

Human tissue-derived preclinical models may reduce extrapolation of evidence for clinically relevant translational potential $(16,18,41)$. Current research directions have provided optimized human tissue-derived cell culture models for triple negative molecular subtype of clinical breast cancer that represent a clinically relevant experimental system. The present data on the growth inhibitory efficacy of natural products such as dietary supplements and their constitutive bioactive agents validates an experimental approach to evaluate natural products as testable alternatives for chemo-endocrine therapy-resistant breast cancer.

Acquired tumor resistance to conventional chemo-endocrine therapy and selection of a survival pathway-targeted therapy are frequently observed phenomena responsible for compromised therapeutic efficacy and disease progression. It occurs predominantly due to the emergence of drug-resistant 
stem cells $(6,40,41)$. Data presented on the isolation and characterization of DOX-resistant triple negative MDA-MB-231 cells validate a potential model for stem cell-targeted therapy.

In the present context, it needs to be recognized that the cell culture models derived from established cell lines provide only limited clinical relevance. Therefore, future research directions focusing on ex vivo approaches for patient-derived xenografts from therapy-resistant breast cancer subtypes, reliable stem cell models from clinical samples, and cellular/ molecular approaches to evaluate lead compound efficacy targeted towards cancer stem cells are expected to provide valuable means to attain better clinical translatability.

\section{Acknowledgements}

Productive collaborations with former colleagues Drs Meena Katdare, Kotha Subbaramaiah, Andrew Dannenberg, Daniel Sepkovic, H. Leon Bradlow, George Y.C. Wong and Michael P. Osborne are gratefully acknowledged. Research Programs in the author's laboratory have been funded by the National Cancer Institute Grants/Contracts (CA-44741, CA-29502 and CN-75029-63), Department of Defense Breast Cancer Research Program (IDEA Award DAMD17-94-J-4208), and by philanthropic funds to the Strang Cancer Prevention Center and to the American Foundation for Chinese Medicine.

\section{References}

1. American Cancer Society - Facts and Figures. American Cancer Society, Atlanta, 2016.

2. Sørlie T, Perou CM, Tibshirani R, Aas T, Geisler S, Johnsen H, Hastie T, Eisen MB, van de Rijn M and Jeffrey SS: Gene expression patterns of breast carcinomas distinguish tumor subclasses with clinical implications. Proc Natl Acad Sci USA 98: 10869-10874, 2001.

3. Baselga J and Swain SM: Novel anti-cancer agents: Revisiting ERBB2 and discovering ERBB3. Nat Rev Cancer 9: 463-475, 2009.

4. Dinh P, Satirou C and Piccart MJ: The evaluation of treatment strategies: Aiming at the target16: S10-S16, 2007.

5. Anders CK, Winer EP, Ford JM, Dent R, Silver DP, Sledge GW and Carey LA: Poly (ADP-ribose) inhibition: Targeted therapy for triple negative breast cancer. Clin Cancer Res 16: 4702-4710, 2010

6. Dean M, Fojo T and Bates S: Tumor stem cells and drug resistance. Nat Rev Cancer 5: 275-284, 2005.

7. Tindle HA, Davis RB, Phillips RL and Eisenburg DM: Trends in the use of complementary and alternative medicines by US adults: 1997-2002. Altern Ther Health Med 11: 42-49, 2005.

8. Molassiotis A, Scott JA, Kearney N, Pud D, Magri M, Selvekerova S, Bruyns I, Fernadez-Ortega P, Panteli V, Margulies A, Gudmundsdottir G, et al: Complementary and alternative medicine use in breast cancer patient in Europe. Support Care Cancer 14: 206-267, 2006.

9. Helyer LK, Chin S, Chui BK, Fitzgerald B, Verma S, Rakovitch E, Dranitsaris $\mathrm{G}$ and Clemons $\mathrm{M}$ : The use of complementary and alternative medicine among patients with locally advanced breast cancer. A descriptive study. BMC Cancer 6: 39-46, 2006.

10. Mukherjee B, Telang N and Wong GYC: Growth inhibition of estrogen receptor positive human breast cancer cells by Taheebo from the inner bark of Tabebuia avellandae tree. Int $\mathrm{J}$ Mol Med 24: 253-260, 2009.

11. Li G, Sepkovic DW, Bradlow HL, Telang NT and Wong GYC: Lycium barbarum inhibits growth of estrogen receptor positive human breast cancer cells by favorably altering estradiol metabolism. Nutr Cancer 61: 408-414, 2009.

12. Telang NT, Li G, Sepkovic DW, Bradlow HL and Wong GYC: Anti-proliferative effects of Chinese herb Cornus officinalis in a cell culture model for estrogen receptor positive clinical breast cancer. Mol Med Rep 5: 22-28, 2012.
13. Telang N, Li G, Sepkovic D, Bradlow HL and Wong GYC: Comparative efficacy of extracts form Lycium barbarum bark and fruit on estrogen receptor positive human mammary carcinoma MCF-7 cells. Nutr Cancer 66: 278-284, 2014.

14. Telang N, Li G, Katdare M, Sepkovic D, Bradlow L and Wong GYC: Inhibitory effects of Chinese nutritional herbs in isogenic breast carcinoma cells with modulated estrogen receptor function. Oncol Lett 12: 3949-3957, 2016.

15. Telang NT, Li G, Katdare M, Sepkovic DW, Bradlow HL and Wong GYC: The nutritional herb Epimedium grandiflorum inhibits the growth in a model for the Luminal A molecular subtype of breast cancer. Oncol Lett 13: 2477-2482, 2017.

16. Katdare M, Osborne MP and Telang NT: Novel cell culture models for prevention of human breast cancer. Int J Oncol 22: 505-519, 2003 (Review).

17. Katdare M, Osborne MP and Telang NT: Soy isoflavone genestein modulates cell cycle progression and induces apoptosis in HER-2/neu oncogene expressing human breast epithelial cells. Int J Oncol 21: 809-816, 2002.

18. Telang $\mathrm{N}$ and Katdare $\mathrm{M}$ : Epithelial cell culture models for prevention and therapy of clinical breast cancer (Review). Oncol Lett 3: 744-750, 2012.

19. Telang NT, Nair HB and Wong GYC: Efficacy of Tabebuia avellandae extract on a cell culture model for triple negative breast cancer. Cancer Res 74 (Suppl.): SABCS Abstract no. P5-14-02, 2014.

20. Telang NT, Nair HB and Wong GYC: Effect of Cornus officinalis (CO) on a model for triple negative breast cancer. Cancer Res 75 (Suppl.): SABCS Abstract no. P3-09-04, 2015.

21. Telang N, Nair HB and Wong GYC: Efficacy of Dipsacus asperoides (DA) in a model for triple negative breast cancer. Cancer Res. 76 (Suppl.): SABCS Abstract no. P4-13-04, 2016.

22. Stampfer MR and Bartley JC: Induction of transformation and continuous cell lines from normal human mammary epithelial cells after exposure to Benzo $(\alpha)$ pyrene. Proc Natl Acad Sci USA 82: 2394-2398, 1985.

23. Neve RM, Chin K, Fridlyand J, Yeh J, Baehner FL, Fevr T, Clark L, Bayani N, Coppe JP, Tong F, Speed T, et al: A collection of breast cancer cell lines for the study of functionally distinct cancer subtypes. Cancer Cell 10: 515-527, 2006.

24. Subik K, Lee JF, Baxter L, Strzepek T, Costello D, Crowley P, Xing L, Hung MC, Bonfiglio T, Hicks DG and Tang P: Expression patterns of ER, PR, HER-2, CK5/6, Ki67 and AR by immuno-histochemical analysis in breast cancer cell lines. Breast Cancer 4: 35-41, 2010.

25. Hudis CA and Gianni L: Triple negative breast cancer: An unmet medical need. Oncologist 16: 1-11, 2011

26. Lin NU, Vanderplas A, Hughes ME, Theriault RL, Edge SB, Wong YN, Blayney DW, Niland JC, Winer EP and Weeks JC: Clinicopathological features, patterns of recurrence and survival amongst women with triple negative breast cancer in National Comprehensive Cancer Network. Cancer 118: 5463-5472, 2012.

27. Gorsky DH: Integrative oncology: Really the best of both worlds? Nat Rev Cancer 14: 692-700, 2014.

28. Huang MT, Ho CT, Wang ZY, Ferraro T, Lou YR, Stauber K, Ma W, Georgiadis C, Laskin JD and Conney AH: Inhibition of skin tumorigenesis by rosemary and its constituents carnosol and ursolic acid. Cancer Res 54: 701-708, 1994.

29. Subbaramaiah K, Chung WJ, Michaluart P, Telang N, Tanabe T, Inoue H, Jang M, Pezzuto JM and Dannenberg AD: Resveratrol inhibits Cyclo-oxygenase transcription and activity in phorbol ester treated human mammary epithelial cells. J Biol Chem 273: 21875-21882, 1998.

30. Subbaramaiah K, Michaluart P, Sporn MB and Dannenberg AJ: Ursolic acid inhibits cyclo-oxygenase-2 transcription in human mammary epithelial cells. Cancer Res 60: 2399-2404, 2000.

31. Subbaramaiah K, Cole PA and Dannenberg AJ: Retinoids and carnasol suppress cyclo-oxygenase- 2 transcription by CREB binding protein/p300-dependent and independent mechanisms. Cancer Res 62: 2522-2530, 2002.

32. Cox LA, Chen G and Lee EY: Tumor suppressor genes and their role in breast cancer. Breast Cancer Res Treat 32: 19-38, 1994.

33. Burkhart DL and Sage J: Cellular mechanisms of tumor suppression by the retinoblastoma gene. Nat Rev Cancer 8: 671-682, 2008 .

34. Bosco EE and Knudson ES: RB in breast cancer: At the crossroads of tumorigenesis and treatment. Cell Cycle 6: 667-671, 2007.

35. Van Arsdale T, Boschoff C, Arndt KT and Abraham RT: Molecular pathways: Targeting the Cyclin D-CDK 4/6 axis for cancer treatment. Clin Cancer Res 21: 2905-2910, 2015. 
36. Muller PAJ and Vousden KH: Mutant p53 in cancer: New functions and therapeutic opportunities. Cancer Cell 25: 304-317, 2014.

37. Ye L, Jia Y, Ji KE, Sanders AJ, Xue K, Ji J, Mason MD and Jiang WG: Traditional Chinese medicine in the prevention and treatment of breast cancer and cancer metastasis. Oncol Lett 10 1240-1250, 2015.

38. Stingl J and Caldas C: Molecular heterogeneity of breast carcinoma and the cancer stem cell hypothesis. Nat Rev Cancer 7: 791-799, 2007.

39. Lobo NA, Shimono Y, Quan D and Clarke MF: The biology of cancer stem cells. Annu Rev Cell Dev Biol 23: 675-699, 2007.

40. Patel SA, Ndabahaliye A, Lim PK, Milton R and Rameshwar P: Challenges in the development of future treatments for breast cancer stem cells. Breast Cancer 2: 1-11, 2010.

41. Telang N: Putative cancer initiating stem cells in cell culture models for molecular subtypes of clinical breast cancer. Oncol Lett 10: 3840-3846, 2015.

42. Telang N: Anti-inflammatory drug resistance selects putative cancer stem cells in a cellular model for genetically predisposed colon cancer. Oncol Lett (In press).
43. Zhang F, Song C, Ma Y, Tang L, Xu Y and Wang H: Effect of fibroblasts on breast cancer cell mammosphere formation and regulation of stem cell related gene expression. Int J Mol Med 28: 365-371, 2011.

44. Takahashi K, Tanabe K, Ohnuki M, Narita M, Ichisaka T, Tomoda $\mathrm{K}$ and Yamanaka S: Induction of pluripotent stem cells from adult human fibroblasts by defined factors. Cell 131: 861-872, 2007.

45. Park IH, Zhao R, West JA, Yabuuci A, Huo H, Ince TA, Lerou PH, Lansch MW and Daley GQ: Reprograming of human somatic cells to pluripotency with defined factors. Nature 451: 141-146, 2008.

46. Yu J, Hu K, Smuga-Otto K, Tian S, Stewart R, Slukvin II and Thomson JA: Human induced pluripotent stem cells free of vector and transgene sequences. Science 324: 797-800, 2009.

47. Ishida R, Koyanagi-Aoi M, Oshima N, Kakeji Y and Aoi T: The tissue reconstructing ability of colon CSCs is enhanced by FK506 and suppressed by GSK3 inhibition. Mol Cancer Res: July 14, 2017 (Epub ahead of print). https://doi.org/10.1158/1541-7786. MCR-17-0071. 